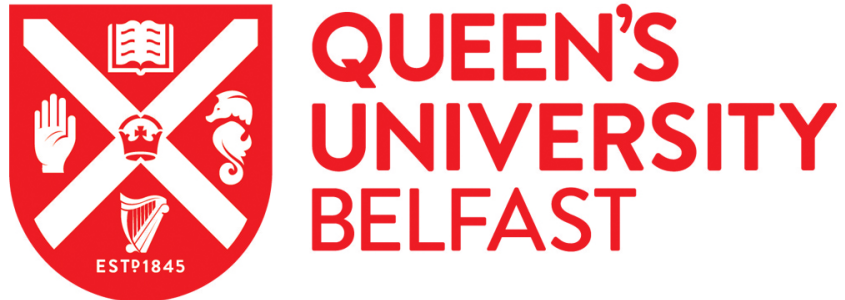

\section{Stable GeV lon Beam Acceleration from Thin Foils by Circularly Polarized Laser Pulses}

Qiao, B., Zepf, M., Borghesi, M., \& Geissler, M. (2009). Stable GeV Ion Beam Acceleration from Thin Foils by Circularly Polarized Laser Pulses. Physical Review Letters, 102(14), [145002].

https://doi.org/10.1103/PhysRevLett.102.145002

\section{Published in:}

Physical Review Letters

Document Version:

Publisher's PDF, also known as Version of record

Queen's University Belfast - Research Portal:

Link to publication record in Queen's University Belfast Research Portal

Publisher rights

(C) 2009 The American Physical Society

\section{General rights}

Copyright for the publications made accessible via the Queen's University Belfast Research Portal is retained by the author(s) and / or other copyright owners and it is a condition of accessing these publications that users recognise and abide by the legal requirements associated with these rights.

Take down policy

The Research Portal is Queen's institutional repository that provides access to Queen's research output. Every effort has been made to ensure that content in the Research Portal does not infringe any person's rights, or applicable UK laws. If you discover content in the Research Portal that you believe breaches copyright or violates any law, please contact openaccess@qub.ac.uk. 


\title{
Stable GeV Ion-Beam Acceleration from Thin Foils by Circularly Polarized Laser Pulses
}

\author{
B. Qiao, M. Zepf, M. Borghesi, and M. Geissler \\ Center for Plasma Physics, Department of Physics and Astronomy, Queen's University Belfast, Belfast BT7 1NN, United Kingdom
}

(Received 14 November 2008; published 8 April 2009)

\begin{abstract}
A stable relativistic ion acceleration regime for thin foils irradiated by circularly polarized laser pulses is suggested. In this regime, the "light-sail" stage of radiation pressure acceleration for ions is smoothly connected with the initial relativistic "hole-boring" stage, and a defined relationship between laser intensity $I_{0}$, foil density $n_{0}$, and thickness $l_{0}$ should be satisfied. For foils with a wide range of $n_{0}$, the required $I_{0}$ and $l_{0}$ for the regime are theoretically estimated and verified with the particle-in-cell code ILLUMINATION. It is shown for the first time by $2 \mathrm{D}$ simulations that high-density monoenergetic ion beams with energy above $\mathrm{GeV} / \mathrm{u}$ and divergence of $10^{\circ}$ are produced by circularly polarized lasers at intensities of $10^{22} \mathrm{~W} / \mathrm{cm}^{2}$, which are within reach of current laser systems.
\end{abstract}

DOI: 10.1103/PhysRevLett.102.145002

Since the first observations [1] of energetic ion beams from laser-irradiated foil targets, laser-driven ion acceleration has been a rapidly progressing field of great interest. This interest is driven by the ion beams' potential for many groundbreaking applications in scientific, technological, and medical areas [2]. Ion beams with energies up to several tens of $\mathrm{MeV}$ have been obtained in experiments, and the mechanism is generally attributed to target normal sheath acceleration (TNSA) [1,3,4]. However, these beams are typically characterized by low particle density, large divergence, and almost $100 \%$ energy spread. A different scheme of efficient ion acceleration by the radiation pressure of ultraintense lasers has been highlighted by particlein-cell (PIC) simulations [5]. However, with linearly polarized pulses, it requires extremely high intensities at $I>$ $10^{23} \mathrm{~W} / \mathrm{cm}^{2}$, not currently achievable in experiments.

Recently, theoretical attention has focused on the use of circularly polarized (CP) laser pulses to accelerate highdensity ion bunches at the front surface of thin foils [6-9]. For CP pulses, the ponderomotive force has no oscillating component; hence, electrons are steadily pushed forward, inducing a charge separation field which can accelerate ions. The absence of hot electrons circulating through the target suppresses rapid foil decompression and allows radiation pressure acceleration (RPA) to dominate at more moderate intensities. Currently, all these studies have followed the acceleration for a limited time duration and have been mostly based on one-dimensional simulations. In the multidimensional case, the acceleration process is more complex as various effects would occur. In general, since the foil is not uniformly irradiated in the transverse dimension, it deforms quickly and becomes transparent resulting in transverse heating of the electrons. As a result, ions can not be stably accelerated to higher energy due to transverse Rayleigh-Taylor-like or Weibel instabilities $[7,10,11]$. To our best knowledge, no multidimensional simulation has yet shown that ion beams can be stably accelerated to hundreds of $\mathrm{MeV}$ per nucleon by $\mathrm{CP}$ laser pulses.
PACS numbers: 52.38.Kd, 29.25.-t, 52.50.Jm, 52.65.Rr

In this Letter, we present a regime of stable relativistic ion acceleration from thin foils by intense CP laser pulses. In contrast to previously discussed schemes, high-density $\mathrm{GeV}$ ion beams are here obtained by sustained, stable acceleration, and therefore the required intensity is within reach of current laser systems. A theoretical model is established, based on which the matching conditions of laser intensity $I_{0}$, foil density $n_{0}$, and thickness $l_{0}$ for this regime are estimated. For a wide range of $n_{0}$, twodimensional (2D) PIC simulations have been run to verify the model. High-density ion beams with energy above $1 \mathrm{GeV} / \mathrm{u}$ and divergence about $10^{\circ}$ are produced by $\mathrm{CP}$ pulses at intensities of the order of $10^{22} \mathrm{~W} / \mathrm{cm}^{2}$.

This stable relativistic ion acceleration regime is composed of two smoothly connected stages: relativistic "holeboring" and "light-sail" RPA. First, CP laser pulse interacts with the foil front surface accelerating and compressing material in a pistonlike manner but with no action on the rear surface-the so-called "hole-boring" stage [6,1214]. Second, if the foil is sufficiently thin, the hole boring reaches its rear surface, the laser pulse punches through and accelerates the compressed ion and electron layers, detached from the foil as a single quasineutral plasma slab. The underlying physics is similar to the "light-sail" concept of space-flight $[15,16]$ - hence, "light-sail" stage. We find that the stability of the acceleration process depends critically on a smooth transition between the two stages. Any significant discontinuity in the acceleration during the transition from "hole-boring" to "light-sail" stages leads to a decompression of the ion bunch and termination of the stable acceleration.

In the "hole-boring" stage, when the $\mathrm{CP}$ laser pulse is incident on the foil front surface, electrons are quickly pushed into the foil by its steady ponderomotive force. The electrons pile up in a compressed layer, leaving behind a charge-separation region and giving rise to a localized spike of electrostatic field which holds them back. Ions are then accelerated by the electrostatic field until catching up with the compressed electrons. At the end of this stage, a 
high-density energetic ion layer (beam) is formed and comoves together with the compressed electron layer. Note that the expression of the radiation pressure for both hole-boring and light-sail phase is $(2 I / c) \times$ $[(1-v / c) /(1+v / c)]$, where $v$ is the ion velocity and $c$ is light speed. In the hole-boring stage, the radiation pressure on the laser-facing ion surface is $2 I / c \times(1-$ $\left.v_{b} / c\right) /\left(1+v_{b} / c\right)$, where $v_{b}$ is the hole-boring velocity. Through momentum and energy conservation, the maximum velocity that ions obtain is [6-9]

$$
\frac{v_{i}}{c}=2 \frac{v_{b}}{c}=2 \sqrt{\frac{I}{m_{i} n_{i} c^{3}}},
$$

where $I$ is laser intensity, $n_{i}$ and $m_{i}$ are ion density and mass. Therefore, the high-density ion layer formed has a velocity distribution ranging from $v_{b}$ to $v_{i}$.

In the "light-sail" stage, the accelerating ion and electron layers are rapidly detached from the foil. Together they constitute a quasineutral plasma slab acting as a reflecting mirror with thickness $l_{s}$ and density $n_{s}$, where $l_{s} \sim n_{s}^{-1 / 2}$. At the transition point between the hole-boring and light-sail stages (i.e., when the laser pulse pushes through the foil), the laser pulse begins to interact with ions traveling at $v_{i}=2 v_{b}$. This causes a sudden jump in the radiation pressure from $(2 I / c)\left[\left(1-v_{b} / c\right) /(1+\right.$ $\left.\left.v_{b} / c\right)\right]$ to $(2 I / c)\left[\left(1-v_{i} / c\right) /\left(1+v_{i} / c\right)\right]$ resulting in a discontinuity in the acceleration. Recent publications [5,7] have shown that this stage can be well modeled by treating the slab as a single object. The ion motion obeys

$$
\frac{d p}{d t}=\frac{2 I}{\sigma m_{i} c^{2}} \frac{\sqrt{1+p^{2}}-p}{\sqrt{1+p^{2}}+p},
$$

where $p=P / m_{i} c$ is the normalized momentum of ions, $\sigma=n_{s} l_{s} \sim n_{0} l_{0}$ is the area density of the slab. In this stage, ions effectively acquire the energy $\left(1-1 / 4 \gamma^{2}\right) \mathcal{E}_{L}$ up to $\mathrm{GeV}$ order by repeated acceleration of the slowest ions at the rear of the ion bunch in the electrostatic sheath field maintained by the laser pressure, where $\mathcal{E}_{L}=I S \tau_{L}$ is the laser pulse energy, $\tau_{L}$ and $S$ are its pulse duration and transverse area, and $\gamma=\left(1+p^{2}\right)^{1 / 2}$ is the Lorentz factor.

To achieve stable acceleration for ion beams, one must drive the hole-boring acceleration hard enough to obtain relativistic ions at this stage before the transition point. This affects the acceleration in two key ways. On the one hand, the difference in the radiation pressure that ions experience between the two stages will be greatly reduced as both $v_{b}$ and $v_{i}$ are close to $c$, resulting in a smooth connection with no significant discontinuity. On the other hand, the transverse instabilities (Rayleigh-Taylor, Weibel and other multidimensional effects) of the ion beam in the "light-sail" RPA stage will be much suppressed as their growth becomes $\gamma$ times slower in the moving frame due to the relativistic effect. While Eq. (1) is not relativistically correct, we can use it to estimate the laser intensity required to produce relativistic ions in the hole-boring stage as

$$
\frac{I_{0}}{m_{i} n_{i} c^{3}} \approx \frac{1}{4} .
$$

For protons $\left(m_{i} / m_{e}=1836\right)$, the intensity is $I_{0} \gtrsim$ $\left(n_{0} / n_{c}\right) \times 6.3 \times 10^{20} \times(1 \mu \mathrm{m} / \lambda)^{2} \mathrm{~W} / \mathrm{cm}^{2}$, where $n_{0}$ is initial foil density and $n_{i}=n_{0}$ is assumed, $\lambda$ is laser wavelength in microns, $n_{c}=m_{e} \omega^{2} / 4 \pi e^{2}$ is the critical density with $e$ and $m_{e}$ electron charge and mass, $\omega$ is laser frequency. As will be shown later using PIC simulations, such intensities are large enough to achieve a smooth transition between "hole-boring" and "light-sail" stages and, consequently, stable acceleration to relativistic energies. Furthermore, if all foil electrons are blown out by the laser ponderomotive force, no equilibrium for compressed electrons exists, and ions cannot be accelerated. Thus, the maximum charge-separation field $E_{\|, \max }=4 \pi e n_{0} l_{0}$ should be larger than the ponderomotive force $\mathbf{v} \times \mathbf{B}_{L} \sim$ $E_{L}$. This gives the condition

$$
\frac{l_{0}}{\lambda} \gtrsim \frac{1}{2 \pi} \sqrt{\frac{n_{c} m_{i}}{n_{i} m_{e}}} \sqrt{\frac{I}{m_{i} n_{i} c^{3}}} .
$$
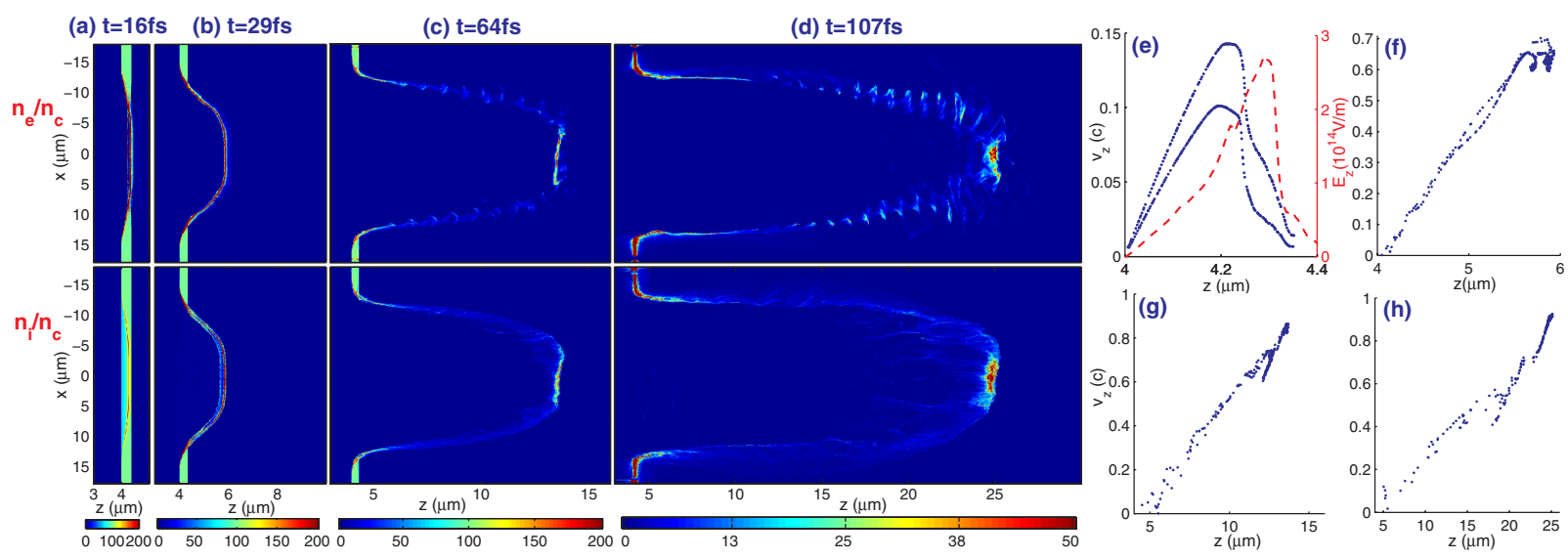

FIG. 1 (color online). Electron density $n_{e} / n_{c}$ and ion density $n_{i} / n_{c}$ in the $(z, x)$ plane at (a) $t=16$, (b) 29 , (c) 64 , and (d) 107 fs for a foil of $n_{0}=100 n_{c}$ and $l_{0}=0.35 \mu \mathrm{m}$ irradiated by a CP laser pulse at $I_{0}=6.3 \times 10^{22} \mathrm{~W} / \mathrm{cm}^{2}$. (e)-(h) are the corresponding ion phase space plots $\left(z, v_{z}\right)$. 
Choosing $I=I_{0}$ and $n_{i}=n_{0}$, we obtain that the foil thickness should satisfy $l_{0} \gtrsim 3.41 \sqrt{n_{c} / n_{0}} \lambda$.

To summarize, ion stable acceleration to relativistic energies is expected when Eq. (3) is satisfied for smooth connection of the two stages and Eq. (4) for no electron blowout.

In order to examine the theoretical model, we carry out 2D PIC simulations with the code ILLUMINATION [17]. Foils with a wide range of densities $n_{0}$ from 20 to $100 n_{c}$ have been verified. In the simulations, 8000 cells along laser axis $z$ and 5000 cells transversely are used forming a $32 \mu \mathrm{m} \times 40 \mu \mathrm{m}$ box. Each cell is filled with 122 quasiparticles. The boundary conditions are absorbing for both electromagnetic waves and quasiparticles. We assume the foils are fully ionized by strong laser field into protons and electrons $\left(m_{i} / m_{e}=1836\right)$.

For the foil with density $n_{0}=100 n_{c}$, where $n_{c}=1.1 \times$ $10^{21} \mathrm{~cm}^{-3}$ assuming $\lambda=1 \mu \mathrm{m}$, we choose a CP laser pulse at $I_{0}=6.3 \times 10^{22} \mathrm{~W} / \mathrm{cm}^{2}$ according to the theoretical estimate [Eq. (3)]. The foil thickness is $l_{0}=0.35 \mu \mathrm{m}$ satisfying Eq. (4); i.e., the areal density is $\sigma_{0}=$ $3.8 \times 10^{18} \mathrm{~cm}^{-2}$. The laser pulse is temporally Gaussian and transversely fourth-order super-Gaussian, $I \sim$ $I_{0} \exp \left[-\left(r / r_{0}\right)^{4}\right] \exp \left\{-\left[\left(t-t_{0}\right) / \tau_{L}\right]^{2}\right\}$, where $r_{0}=10 \mu \mathrm{m}$, $t_{0}=33$ fs and $\tau_{L}=38$ fs are taken. Figure 1 plots electron density $n_{e} / n_{c}$, ion density $n_{i} / n_{c}$, and phase space $(z$, $v_{z} / c$ ) at $t=16,29,64$, and $107 \mathrm{fs}$. Part (a) corresponds to the initial "hole-boring" stage. We see that the compressed electron layer is formed in the region of the laser focal spot by the steady ponderomotive push, inducing a strong charge-separation field $E_{z}$. The profile of $E_{z}$ is shown by the dashed line in Fig. 1(e), which increases with the distance from the foil front surface up to the position of the compressed electron layer where reaching the peak value of $2.8 \times 10^{14} \mathrm{~V} / \mathrm{m}$ and steeply decreases in the layer. Then ions are rapidly accelerated by $E_{z}$ and a high-density monoenergetic ion layer with density $\geq 200 n_{c}$ and thickness $\leq 0.1 \mu \mathrm{m}$ is formed [Fig. 1(b)]. The phase space [Fig. 1(f)] shows a distinct "head" where the majority of ions are concentrated, and a "tail" which contains a very low amount of trailing ions. After hole boring, ions are accelerated to the maximum velocity $v_{i} \approx 0.6 c$ [Fig. 1(f)].

Parts (c) and (d) show the accelerating plasma slab fully detached in the "light-sail" stage. The high-density ion and electron layers are detached from the foil quickly and combine together constituting a quasineutral plasma slab with thickness about $0.05 \mu \mathrm{m}$. This accelerating slab moves more and more quickly (up to $0.85 c$ ); thus, it stays opaque and copropagates with laser pulse acting as a relativistic plasma mirror, as seen in Fig. 1(c). Ions effectively acquire energy from the laser via repeated accelerations, the phase space is shown in Fig. 1(g). At $t=107 \mathrm{fs}$, the ion layer is transformed into a high-quality highdensity relativistic ion beam with density about $50 n_{c}$ and peak velocity above $0.9 c$, shown in Fig. 1(d) and 1(h). This relativistic ion beam can propagate over a long distance without exploding since it is kept quasineutral by the relativistic electrons nearby.

Equation (3) can also be fulfilled for lower intensity lasers using a lower density foil. For $n_{0}=30 n_{c}$, the required intensity is $I_{0}=1.89 \times 10^{22} \mathrm{~W} / \mathrm{cm}^{2}$, which is within reach of current laser systems. Keeping $\sigma_{0}$ almost the same according to Eq. (2), we choose $l_{0}=1.0 \mu \mathrm{m}$, i.e., $\sigma_{0}=3.3 \times 10^{18} \mathrm{~cm}^{-2}$. The simulation results show that the stable relativistic acceleration regime is also achieved, and the acceleration dynamics is very similar to the above case of $n_{0}=100 n_{c}$. The ion density and phase space at $t=107$ fs in Figs. 2(a) and 2(d) show a highdensity, high-quality relativistic ion beam with density of $15 n_{c}$ and velocity about $0.9 c$. Dark gray (blue) lines in Figs. 3(a) and 3(b) demonstrate the smooth transition between the hole-boring and "light-sail" acceleration stages. However, if the matching condition Eq. (3) between $n_{0}$ and $I_{0}$ is not satisfied, the acceleration process changes back to the unstable regimes described in Refs. [7-9], where ions cannot be stably accelerated to high energy and the ion beams decompress quickly. Two mismatched examples of the unstable acceleration are also given in Fig. 2, respectively, by increasing $n_{0}$ to $100 n_{c}$ for the same $I_{0}$ and $l_{0}[2(\mathrm{~b})]$ and by decreasing $I_{0}$ of 1 order of magnitude for the same $n_{0}$ and $l_{0}[2(\mathrm{c})]$. In contrast to the stable case, both unstable cases display a discontinuous acceleration profile at the transition point between holeboring and "light-sail" stages [see light gray (green) and black lines in Fig. 3(a)]. The peak longitudinal electric field $E_{z}$ in Fig. 3(b) also clearly shows the significant discontinuity (kink) at the transition points. This discontinuity leads to a sudden increase in the velocity spread of the ions (since only the ions at the rear of the ion beam are

(a)

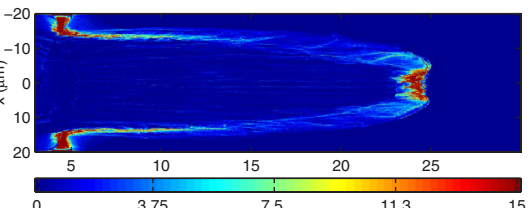

(b)

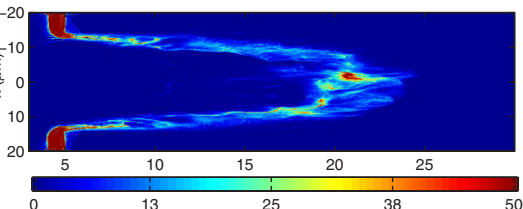

(c) 承
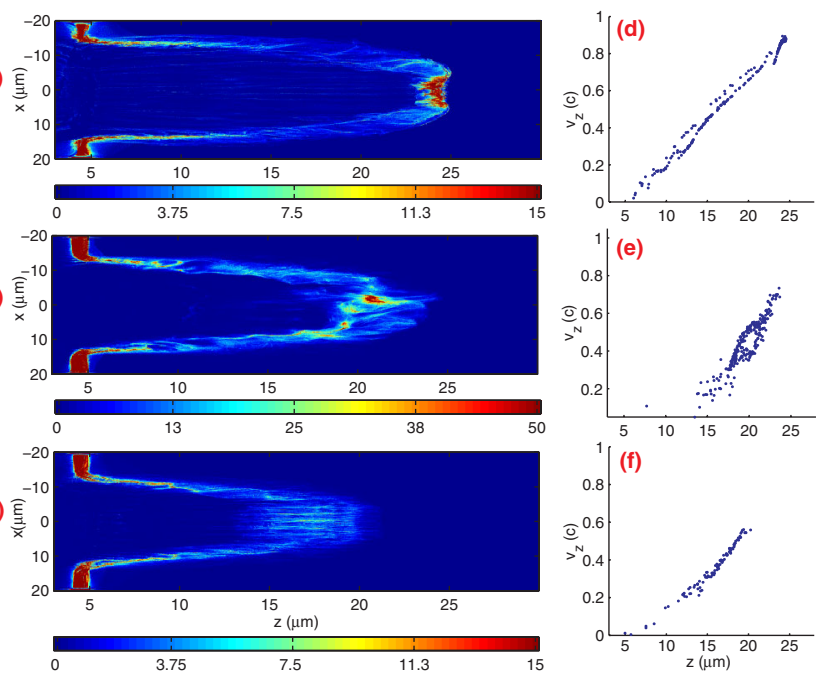

(e)
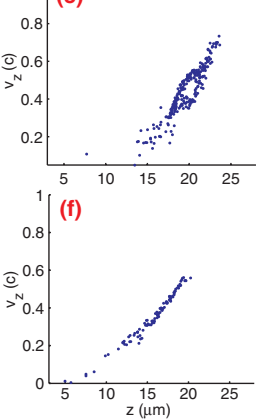

FIG. 2 (color online). Ion density $n_{i} / n_{c}$ (a) and phase space $(z$, $v_{z}$ ) (d) at $t=107 \mathrm{fs}$ for the foil of $n_{0}=30 n_{c}, l_{0}=1.0 \mu \mathrm{m}$ by $\mathrm{CP}$ laser at $I_{0}=1.89 \times 10^{22} \mathrm{~W} / \mathrm{cm}^{2}$. (b) and (e) show an unstable ion acceleration case with the same $I_{0}$ and $l_{0}$ but $n_{0}=$ $100 n_{c}$; (c) and (f) show another unstable case with the same $n_{0}$ and $l_{0}$ but lower $I_{0}=1.89 \times 10^{21} \mathrm{~W} / \mathrm{cm}^{2}$. 

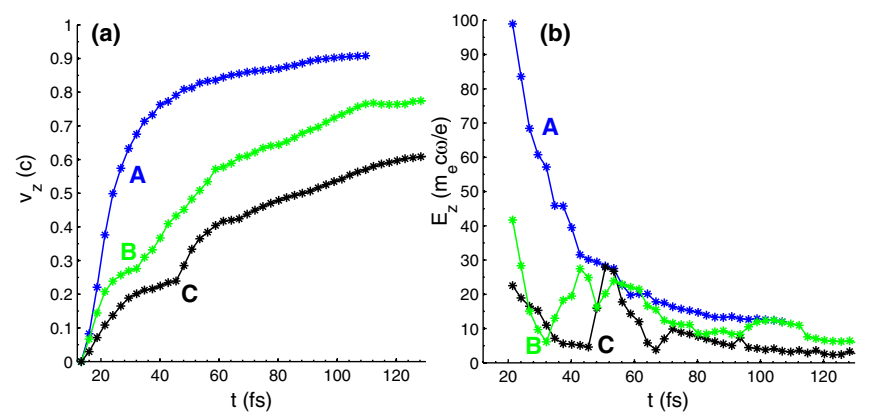

FIG. 3 (color online). (a) The peak velocities $v_{z}$ of ion beams varying with time $t$ the cases, respectively, of Figs. 2(a) [dark gray (blue)], 2(b) [light gray (green)], and 2(c) (black); (b) the corresponding peak value of longitudinal electric fields $E_{z}$ on ion beams. Points "A," "B," and "C" correspond to the transition point from "hole-boring" to "light-sail" stages; "B" and "C" clearly show the discontinuous acceleration profile at the transition of the two stages for the unstable cases.

accelerated at any point in time). The sudden increased velocity spread results in rapid decompression of the ion beam and subsequent rapid instability growth and ultimately transparency. The premature termination of the "light-sail" phase limits the peak energies to much lower values of about 300 and $100 \mathrm{MeV}$ [light gray (green) and black lines in Fig. 4, respectively, give detailed spectra].

The energy spectra of $\mathrm{GeV}$ ion beams at $t=107 \mathrm{fs}$ by stable acceleration are shown in Fig. 4 by gray (red) and dark gray (blue) lines. For the foil of $n_{0}=100 n_{c}$, the ion beam has a peak energy of about $1.35 \mathrm{GeV}$ with the fullwidth-at-half-Maximum (FWHM) range from 1.2 to $1.4 \mathrm{GeV}$, the maximum divergence angle $\theta_{\max }$ is about $10^{\circ}$, the number of ions in the beam is $\mathcal{N}_{i}=1.1 \times 10^{12}$ (beam density $50 n_{c}$ ). For $n_{0}=30 n_{c}$, the peak energy is $1.0 \mathrm{GeV}$ with the FWHM range from 0.86 to $1.05 \mathrm{GeV}$ and $\theta_{\max } \approx 11^{\circ}, \mathcal{N}_{i}$ is about $1.3 \times 10^{12}$ (density $15 n_{c}$ ). For both cases, the efficiency of energy transfer from the laser pulses to ions is greater than $\eta=50 \%$. For the foils with a wide range of densities from 20 and $100 n_{c}$, our simulations verify that the stable relativistic ion acceleration can all be

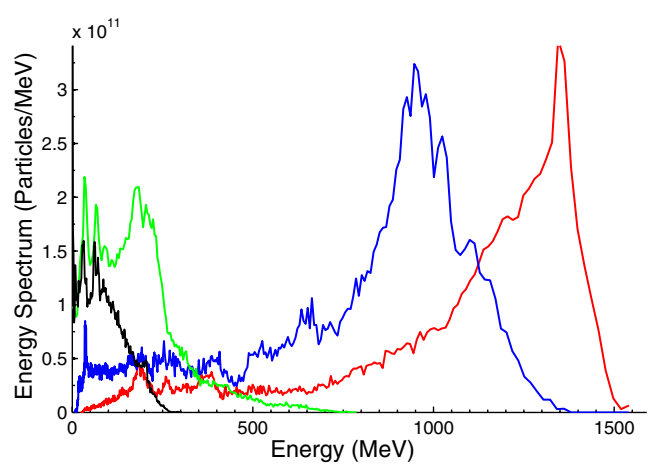

FIG. 4 (color online). The energy spectra of ions in the region of $z>10 \mu \mathrm{m}$ and $|x|<10 \mu \mathrm{m}$ at $t=107 \mathrm{fs}$ for the cases, respectively, of Figs. 1 [gray (red)] and 2(a) [dark gray (blue)], 2(b) [light gray (green)], and 2(c) (black). achieved if Eqs. (3) and (4) are satisfied. Note that the spectra at earlier times are more sharply peaked than final spectra shown here. From our simulations, it appears likely that different pulse profiles and larger laser spots could be used to achieve more narrowly peaked spectra at $\mathrm{GeV}$ energies.

In conclusion, a new regime of stable relativistic ion acceleration from thin foils by CP laser pulses has been proposed. Two-dimensional PIC simulations show that high-density relativistic ion beams with energy above $\mathrm{GeV} / \mathrm{u}$ and beam divergence about $10^{\circ}$ are obtained at intensities within reach of current laser systems (the order of $10^{22} \mathrm{~W} / \mathrm{cm}^{2}$ ). Note that the acceleration time here is limited by the computer resources. If a longer acceleration time is tracked, the ion beams could reach even higher energy. For a laser pulse with finite duration, the upper limit of the ion energy can be estimated from Eq. (2) as $2 \eta^{2} \mathcal{E}_{L}^{2} /\left(2 \eta \mathcal{E}_{L}+\mathcal{N}_{i} m_{i} c^{2}\right) \mathcal{N}_{i}$. For our simulation parameters, these limit the ion energy to about $25 \mathrm{GeV}$. However, one would expect the ion beam to decompress due to spectral broadening at late times, which thus prevents the theoretical maximum energy from being reached. To test this regime experimentally, we suggest to choose a target of relatively low density allowing laser intensities as low as $1 \times 10^{22} \mathrm{~W} / \mathrm{cm}^{2}$ to be used to achieve $\mathrm{GeV} / \mathrm{u}$ ions.

The work here was supported by EPSRC (Grants No. EP/E035728/1 and No. EP/D/06337X/1). M.Z. acknowledges support from the Royal Society. B. Q. acknowledges useful discussion with X.T. He and C.T. Zhou at Center for Applied Physics and Technology, Peking University.

[1] E. L. Clark et al., Phys. Rev. Lett. 84, 670 (2000); A. Maksimchuk et al., ibid. 84, 4108 (2000); R. A. Snavely et al., ibid. 85, 2945 (2000).

[2] M. Borghesi et al., Fusion Sci. Technol. 49, 412 (2006) and references therein.

[3] B. M. Hegelich et al., Nature (London) 439, 441 (2006); H. Schwoerer et al., Nature (London) 439, 445 (2006).

[4] L. Yin et al., Phys. Plasmas 14, 056706 (2007).

[5] T. Esirkepov et al., Phys. Rev. Lett. 92, 175003 (2004).

[6] A. Macchi et al., Phys. Rev. Lett. 94, 165003 (2005).

[7] A. P. L. Robinson et al., New J. Phys. 10, 013021 (2008).

[8] X. Q. Yan et al., Phys. Rev. Lett. 100, 135003 (2008).

[9] X. M. Zhang et al., Phys. Plasmas 14, 073101 (2007).

[10] F. Pegoraro and S. V. Bulanov, Phys. Rev. Lett. 99, 065002 (2007).

[11] M. Chen et al., Phys. Plasmas 15, 113103 (2008).

[12] P. Gibbon, Phys. Rev. E 72, 026411 (2005).

[13] S. C. Wilks et al., Phys. Rev. Lett. 69, 1383 (1992).

[14] T. Lyseikina et al., Plasma Phys. Controlled Fusion 50, 124033 (2008).

[15] J. F. L. Simmons and C. R. McInnes, Am. J. Phys. 61, 205 (1993).

[16] A. P. L. Robinson et al., Plasma Phys. Controlled Fusion (to be published).

[17] M. Geissler et al., New J. Phys. 8, 186 (2006); M. Geissler et al., ibid. 9, 218 (2007). 